\title{
Ultrasound for Critical Care Physicians: Unraveling a Rapid Drop of Hematocrit
}

\author{
Deepti Baheti, MBBS \\ Pablo Garcia, MD \\ Department of Internal Medicine and LifeBridge Critical Care \\ Sinai Hospital of Baltimore. \\ Baltimore, MD USA
}

An 85-year-old woman was admitted to our hospital with complaints of shortness of breath on exertion. Her medical history was significant for hypertension, pulmonary embolism and stage III chronic kidney disease. She was diagnosed with severe decompensated pulmonary hypertension and started to improve with diuretics. While hospitalized, she suffered an asystolic arrest and was successfully resuscitated. As a result of chest compressions, the patient developed multiple anterior rib fractures. Within a few days of recovering from her cardiac arrest, she was anticoagulated with enoxaparin as a bridge to warfarin for her prior history of pulmonary embolism. Five days after initiation of enoxaparin and warfarin, she was noted to have an acute drop in her hemoglobin from $8 \mathrm{~g} / \mathrm{dl}$ to $5 \mathrm{~g} / \mathrm{dl}$. A thorough physical examination revealed a large area of swelling in her left anterior chest wall. Point-of- care ultrasound was utilized to image this area of swelling centered at the 3rd intercostal space between the midclavicular and anterior axillary line (Figures 1 and 2).

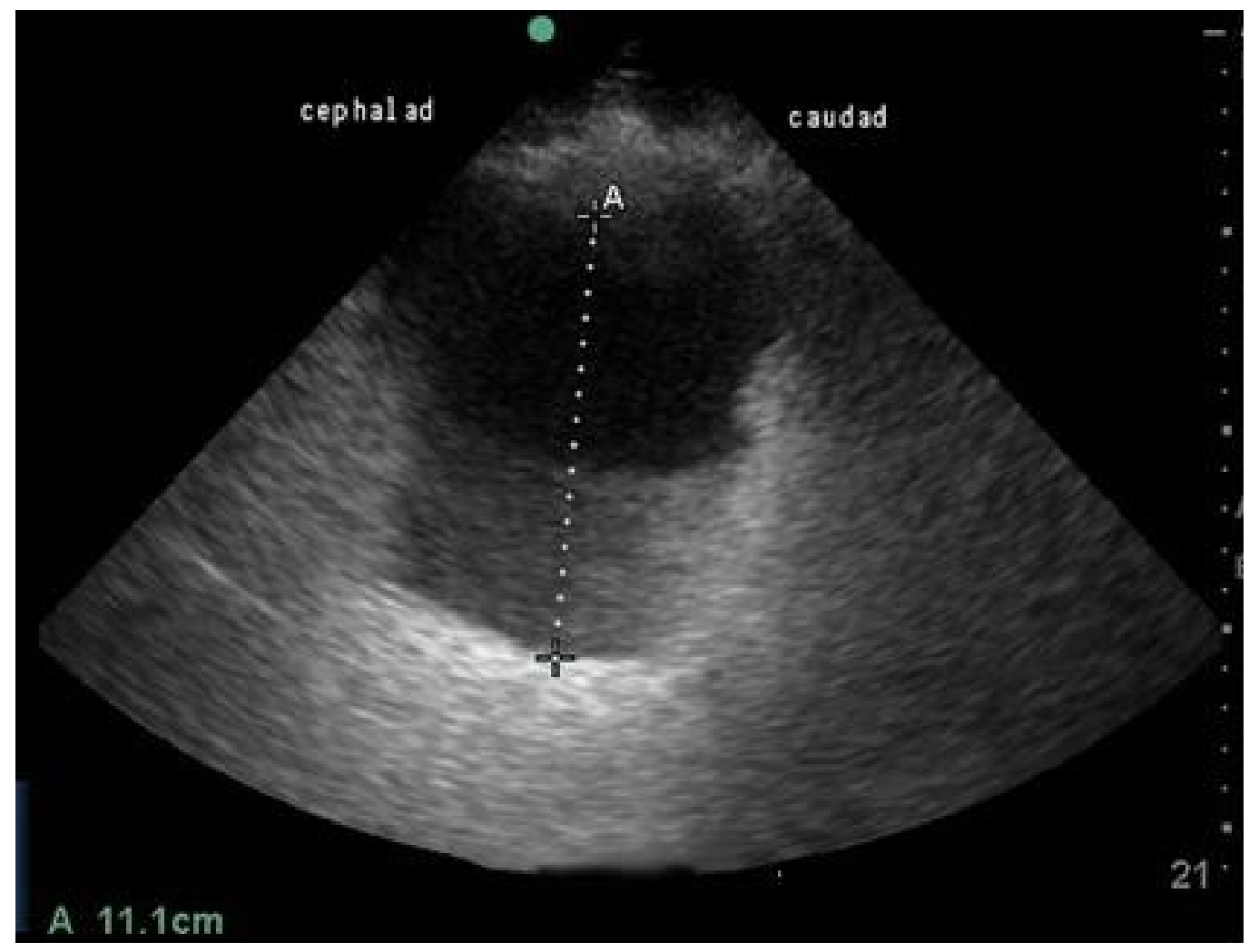

Figure 1. Ultrasound image of the chest wall in the sagittal plane. 


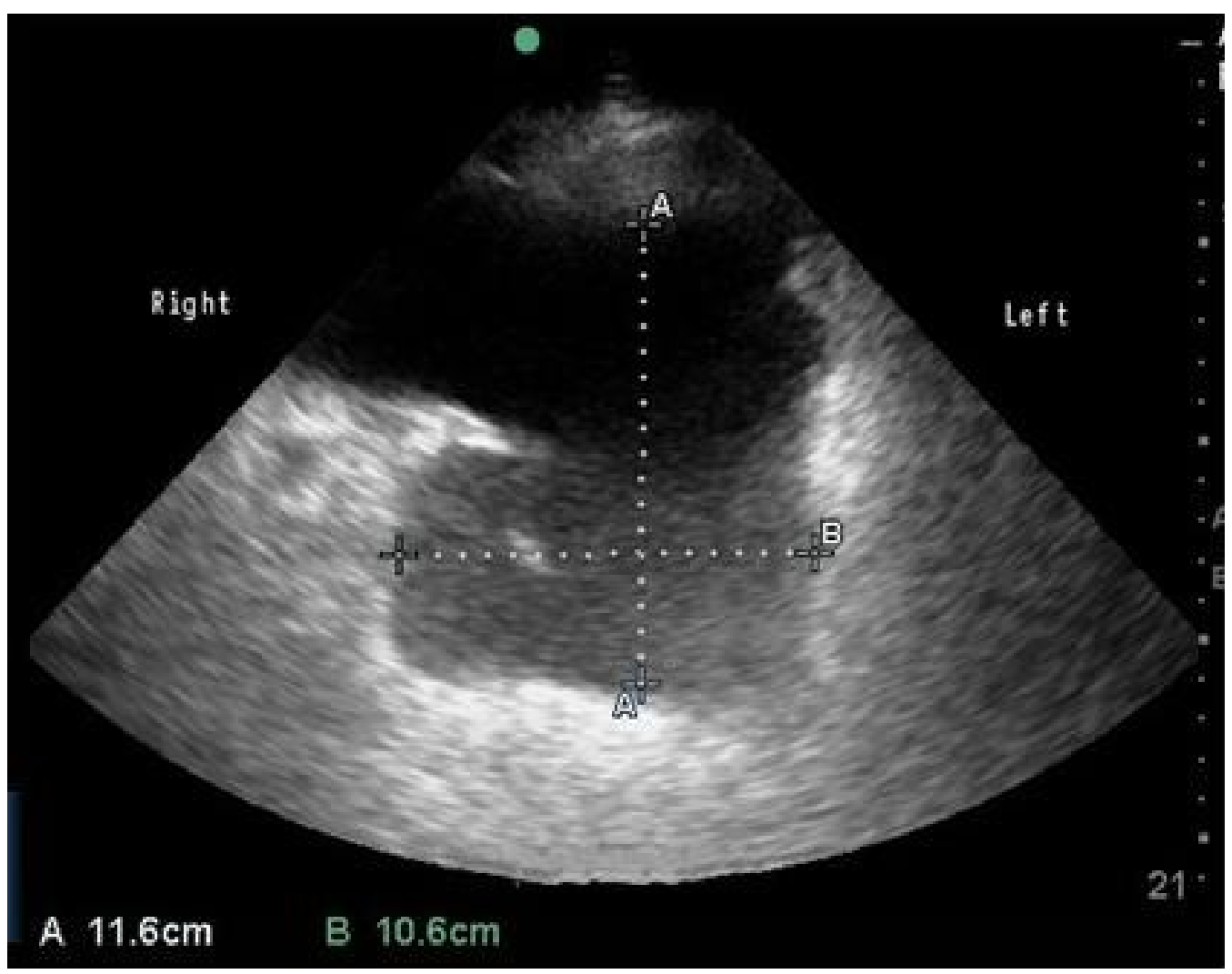

Figure 2. Ultrasound image of the chest wall in the transverse plane.

What is the cause of this patient's acute anemia?

1. Chest wall hematoma

2. Hemolysis

3. Hemoperitoneum

4. Hemothorax 


\section{Correct! \\ 1. Chest wall hematoma}

Point-of-care ultrasound utilized to examine the patient's left chest wall revealed an 11 $\mathrm{cm} \times 13 \mathrm{~cm}$ fluid collection with a distinct change in echo density from anechoic in the superficial half to hyperechoic in the dependent portion. At this point, the ICU team confirmed their suspicion that the swelling of the patient's chest wall was a hematoma with the characteristic ultrasound finding of the hematocrit sign. The hematocrit sign, or linear separation of cellular and liquid components of extravascular blood forming a plasma-blood interface, is identified with point-of care ultrasound and can quickly confirm the diagnosis of hematoma, hemothorax and hemoperitoneum. It is a very useful ultrasound finding to consider when evaluating fluid collections.

\section{Discussion}

The word 'hematocrit' was coined in 1891 by Swedish scientist Magnus Blix and has its roots in the Greek words haima (blood) and kritēs (judge), which when combined means "to separate blood". In the era of modern medicine, the term hematocrit refers to the percentage of whole blood volume occupied by packed erythrocytes. In the early 20th century, hematocrit referred to a device for separating the cellular elements of blood from plasma. Usually, these devices were physician-designed micro-capillary tubes (i.e. Wintrobe hematocrit tube) that were centrifuged to separate the cells from the liquid plasma.

The same separation of cells from plasma can take place when significant volumes of blood pool in the body in extravascular spaces. This gravity-dependent interface between separable layers of differing densities within a space containing at least partially liquid contents can be noted as straight radiographic lines on various imaging studies. The hematocrit effect has been commonly noted on CT and MRI studies in conditions such as retroperitoneal hematoma, subdural hematoma and hemothorax.

With respect to ultrasound imaging, the hematocrit sign refers to the layering effect noted within large collections of extravascular blood when the collection separates into two gravity dependent layers with differing echogenicities. The more dependent layer contains a dense collection of erythrocytes and appears more echogenic than the less dependent layer, primarily made up of plasma. In vitro, with the use of microcapillary tubes and centrifugation, it takes 5-30 minutes for the separation of plasma and cellular components, depending on the device and the tube dimensions. Factors affecting the time it takes for development of hematocrit sign in a hematoma are the patient's coagulation profile, size of hematoma, and patient's ambulatory condition.

To best visualize a chest wall hematoma of significant depth, utilize a low frequency transducer. The initial orientation for thoracic imaging will be with the orientation marker cephalad. A hematoma or other fluid collection will appear anechoic or hypoechoic depending on the fluid's echogenicity and should have an apparent hyperechoic border outlining its shape. Once you have captured a cross section of the fluid collection in the 
sagittal plane, turn probe 90 degrees and identify the dimensions of the fluid collection in a transverse plane.

The volume of blood can be estimated by determining the dimensions of the hematoma in both a sagittal and transverse plane. These two measurements can then be applied to the prolate ellipsoid formula ( $0.52 \mathrm{x}$ anterior-posterior length $\mathrm{x}$ height $\mathrm{x}$ width) to calculate the fluid volume.

\section{Follow-Up}

The patient subsequently underwent endovascular embolization of one of the branches of the costocervical trunk. Despite this intervention, a few days later, she required surgical evacuation and drain placement to prevent the development of compartment syndrome.

\section{References}

1. Day CM, Sodickson A. The art and science of straight lines in radiology. AJR Am J Roentgenol. 2011 Feb;196(2):W166-73. [CrossRef] [PubMed]

2. Tan S, Aronowitz P. Hematocrit effect in bilateral subdural hematomas. J Gen Intern Med. 2013 Feb;28(2):321. [CrossRef] [PubMed]

3. Lubner M, Menias C, Rucker C, Bhalla S, Peterson CM, Wang L, Gratz B. Blood in the belly: CT findings of hemoperitoneum. Radiographics. 2007 Jan-Feb;27(1):10925. [CrossRef] [PubMed]

4. Frizzell JD, Griffin LS, Boivin MA, Saeed Al. A 73-year-old man with left lung "white out". Chest. 2015 Aug;148(2):e38-41. [CrossRef] [PubMed] 